\title{
REGIME DE TRANSPORTE DE POLPA DE MOINHO ISAMILL EM ESCALA DE LABORATÓRIO
}

\author{
D. B. MAZZINGHY ${ }^{1}$, C. L. SCHNEIDER ${ }^{2}$, V. K. ALVES ${ }^{3}$, L. H. REIS ${ }^{3}$, R.A. CHACANA \\ ${ }^{1}$ PPGEM - Universidade Federal de Minas Gerais, ${ }^{2}$ Cetem - Tecnologia Mineral; \\ ${ }^{3}$ Vale S.A. - Centro de Desenvolvimento Mineral; ${ }^{4}$ Asmin \\ cschneid@cetem.gov.br; douglasmazzinghy@ufmg.br; vladmir.alves@vale.com; \\ lucas.reis@vale.com; raul.chacana@asmin.cl
}

Artigo submetido em novembro/2013 e aceito em dezembro/2013

DOI: http://dx.doi.org/10.15628/holos.2014.1746

\section{RESUMO}

O moinho IsaMill tem se mostrado eficiente em etapas de remoagem. Isso ocorre porque os moinhos deste tipo permitem a utilização de corpos moedores muito pequenos em relação a outros tipos de moinhos, notoriamente o moinho tubular de bolas e o VertiMill. Corpos moedores de pequeno diâmetro implicam em eficiência maior na moagem de partículas finas. Neste trabalho foram realizados quatro ensaios de moagem contínuos, em condições diversas, com duas amostras de minério de cobre com granulação distinta. A determinação da distribuição de tempos de residência da fase líquida realizou-se com um ácido como traçador e medidas de $\mathrm{pH}$ no produto do moinho de laboratório de um litro. Os resultados obtidos indicam que o moinho IsaMill de laboratório apresenta um padrão de distribuição de tempo de residência de fluxo pistão e deverá se comportar similarmente a um moinho de barras do ponto de vista de transporte

PALAVRAS-CHAVE: Moagem, IsaMill, Tempo de Residência.

\section{SLURRY TRANSPORT SCHEME OF ISAMILL LABORATORY SCALE}

\begin{abstract}
IsaMill has proven effective in regrind stages. This is because this mill type uses very small grinding balls in relation to other mills, notably the tubular ball mill and VertiMill. Balls of small diameter imply greater efficiency in the grinding of fine particles. In this work were performed four trials of continuous grinding, under various conditions, with two samples of copper ore with
\end{abstract}

distinct particle size distribution. The residence time distribution of the liquid phase was performed with an acid as tracer and $\mathrm{pH}$ measurements. The results indicate that IsaMill lab scale shows a pattern of residence time distribution type piston flow should behave similarly to a rod mill from the transportation viewpoint

KEYWORDS: Grinding, IsaMill, Residence Time. 


\section{INTRODUÇÃO}

Muitos estudos tem mostrado a maior eficiência dos moinhos do tipo IsaMill em moagens de material fino (BOSSE, 1983; KALRA, 1999; STEHR et al., 1987) Isso ocorre porque os moinhos deste tipo permitem a utilização de corpos de moagem muito pequenos em relação a outros tipos de moinhos, notoriamente o moinho tubular de bolas e o VertiMill. Meio moedor pequeno implica em eficiência maior na moagem de partículas finas (JAYASUNDARA et al., 2012; MANKOSA et al., 1986). O mecanismo dos moinhos IsaMill é ilustrado na Figura 1.

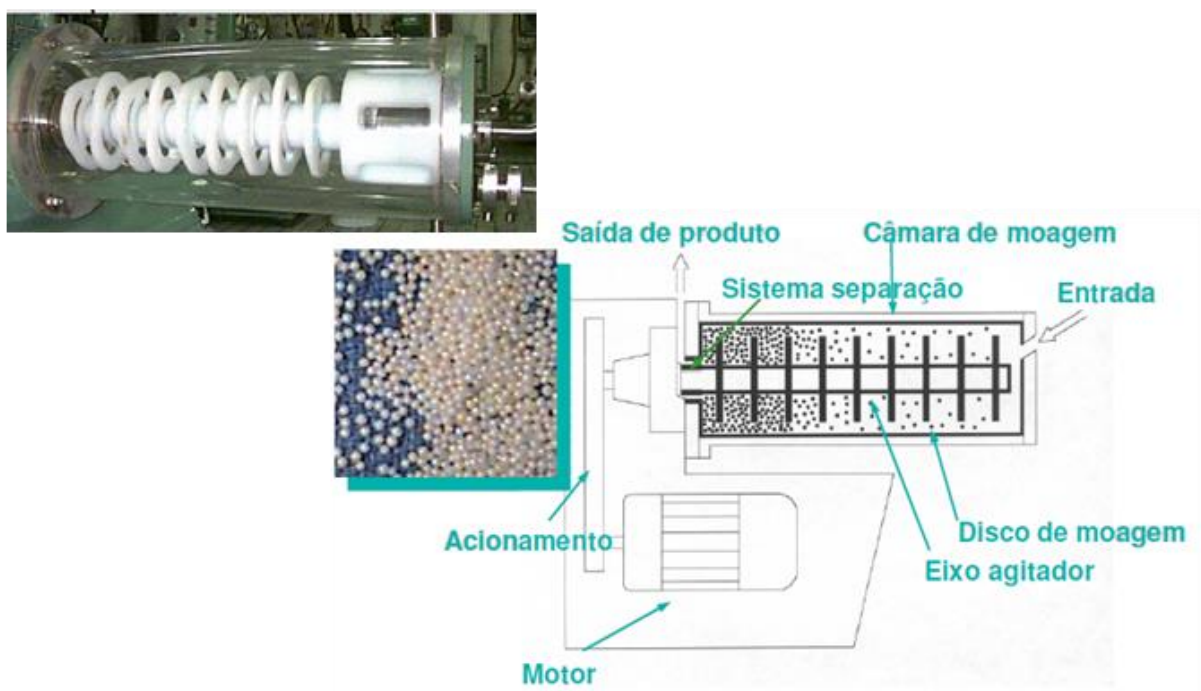

Figura 1. Um moinho IsaMill de laboratório de 1 litro com a carcaça transparente e a descrição dos mecanismos (Cortesia Netzsch)

$O$ rotor no eixo central do moinho é chamado de eixo agitador. Este eixo gira em velocidades relativamente altas, como por exemplo 2000 RPM. A câmera é preenchida com bolas de moagem (ou outro corpo moedor) e água (ou outro líquido). A rotação do eixo produz uma rotação da água (líquido) e consequentemente dos corpos moedores que são empurrados contra a parede interna do cilindro e uns contra os outros. Normalmente, a câmara é preenchida com 70 a 90\% de corpos moedores que em geral são esferas que podem ter tamanhos entre 6 e 0,1 mm. Partículas alimentadas neste ambiente viajam ao longo do cilindro desde o porte de alimentação até o sistema de separação do lado da saída do produto. É importante notar que a saída do produto se dá pelo centro do cilindro e não pela periferia.

As forças centrífugas geradas por este mecanismo são maiores na periferia. Pode-se postular que as partículas maiores disputam espaço na periferia com os corpos moedores enquanto a partículas menores, que experimentam forças menores, tendem a se deslocar pela região mais central da câmara, em direção ao sistema de separação e da saída do produto. À medida que as partículas maiores são quebradas por compressão, os produtos da quebra tendem a dar lugar a novas partículas maiores, e são deslocados em direção ao centro do cilindro.

O mecanismo de quebra por compressão é provavelmente bastante similar ao que ocorre em um moinho de bolas convencional e no VertiMill. Da mesma forma que nos moinhos convencionais, partículas maiores sofrerão esforços maiores e terão taxas de quebra maiores enquanto que partículas menores apresentarão taxas de quebras menores. A diferença principal é 
de escala. Mantendo-se as proporções, uma partícula grossa no IsaMill poderia ser representada por uma partícula de 0,5 mm enquanto que em um moinho convencional uma partícula grossa é dez vezes maior, $5 \mathrm{~mm}$. Porém, no IsaMill seria possível quebrar partículas de 0,5 $\mathrm{mm}$ com taxas semelhantes a que partículas de $5 \mathrm{~mm}$ são quebradas em um moinho convencional.

Estudos recentes indicam que os moinhos do tipo IsaMill possuem os três mecanismos de fragmentação (atrição, abração e impacto), porém em diferentes proporções se comparado com outros tipos de moinhos, e não somente o mecanismo de atrição (KWADE, 1999A; KWADE \& SCHWEDES, 2002; SHINOHARA, 1999). Os mecanismos de quebra dos moinhos do tipo IsaMill são muito discutidos na literatura técnica (HOGG, 1999; KWADE, 1999', YUE \& KLEIN, 2005).

\section{MODELAMENTO}

As questões envolvidas no modelamento de moinhos IsaMill são, neste momento, as seguintes:

- Transporte. Ocorre mistura no sentido longitudinal, como num moinho de bolas? Neste caso a distribuição de tempos de residência será de misturadores perfeitos em série. Alternativamente, se as particulas se misturam no sentido perpendicular ao eixo, como em um moinho de barras, o transporte será de fluxo pistão (plug flow).

- Classificação na saída (exit classification). Se a moagem for descrita por regiões de misturadores perfeitos, provavelmente ocorrerá uma forte classificação na saída, ficando as partículas mais grossas retidas pelo sistema de separação (Figura 1).

- O processo de moagem escalona pelo tempo de residência?

Neste trabalho será inverstigado o mecanismo de transporte, e os resultados levarão ao desenvolvimento de um modelo apropriado para moinhos do tipo IsaMill.

\section{METODOLOGIA}

Quatro ensaios de moagem contínuos, em condições diversas, foram realizados com duas amostras de minério de cobre (dois ensaios cada) com granulação distinta. A Figura 2 apresenta o IsaMill existente no Centro de Desenvolvimento Mineral da Vale S.A.

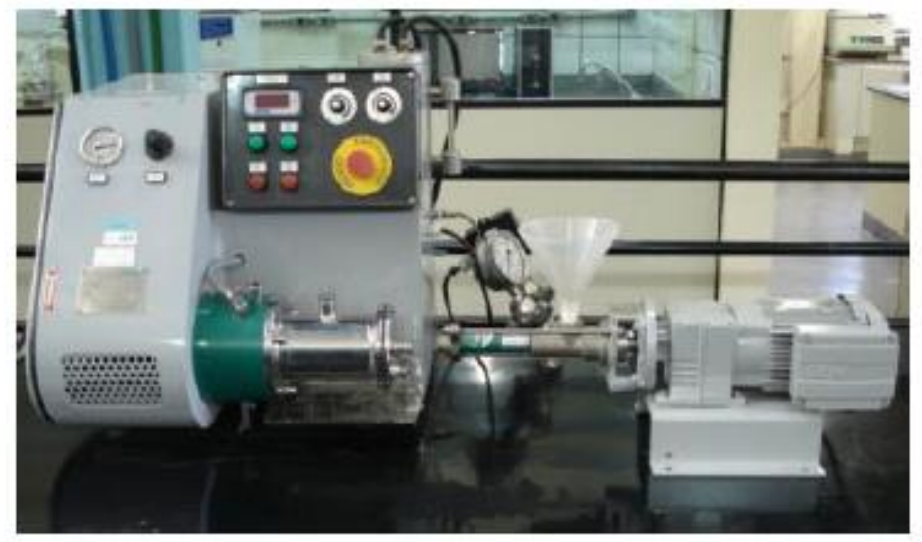

Figura 2. Moinho IsaMill de laboratório de 1 litro do Centro de Desenvolvimento Mineral da Vale S.A. 
Durante o processo de moagem contínua, foi realizado um ensaio de determinação da distribuição de tempos de residência da fase líquida, utilizando-se um ácido como traçador e medidas de $\mathrm{pH}$ no produto do moinho de laboratório de um litro. As condições de todos os ensaios são sumarizadas nas Tabelas 1 e 2.

Tabela 1. Condições comuns aos ensaios de moagem e de determinação da distribuição de tempos de residência

\begin{tabular}{ccc}
\hline Critérios & Unid. & Valor \\
\hline Diâmetro Interno & $\mathrm{mm}$ & 85,15 \\
Comprimento Interno & $\mathrm{mm}$ & 170,05 \\
Volume útil do moinho & $\mathrm{I}$ & 0,80 \\
Densidade real das bolas & $\mathrm{kg} / \mathrm{l}$ & 6,60 \\
Densidade Aparente das bolas & $\mathrm{kg} / \mathrm{l}$ & 4 \\
Diâmetro máximo das bolas & $\mathrm{mm}$ & 2,4 \\
Densidade real dos sólidos & $\mathrm{g} / \mathrm{cm}^{3}$ & 3,37 \\
Densidade aparente dos sólidos & $\mathrm{g} / \mathrm{cm}^{3}$ & 1,51 \\
Tempo de amostragem & $\mathrm{s}$ & 60 \\
Tamanho máximo de partícula & $\mu \mathrm{m}$ & 425 \\
F80 & $\mu \mathrm{m}$ & 89 \\
\hline
\end{tabular}

Tabela 2. Condições dos ensaios de moagem e de determinação da distribuição de tempos de residência

\begin{tabular}{cccccc}
\hline Variável & Unid. & Teste 1 & Teste 2 & Teste 3 & Teste 4 \\
\hline Massa de bolas & $\mathrm{kg}$ & 2,4 & 2,56 & 2,4 & 2,56 \\
Volume de bolas & $\mathrm{I}$ & 0,36 & 0,39 & 0,36 & 0,39 \\
J - Vol. preenchido com bolas & Fração & 0,75 & 0,80 & 0,75 & 0,80 \\
Taxa de sólidos & $\mathrm{kg} / \mathrm{h}$ & 10,72 & 11,28 & 26,49 & 24,17 \\
Vazão de polpa & $\mathrm{l} / \mathrm{h}$ & 28,2 & 28,8 & 28,2 & 26,7 \\
Vazão de água & $\mathrm{l} / \mathrm{h}$ & 25,0 & 25,4 & 20,3 & 19,5 \\
Densidade da polpa & $\mathrm{g} / \mathrm{cm}^{3}$ & 1,27 & 1,28 & 1,66 & 1,64 \\
Concentração de sólidos & $\%$ & 29,99 & 30,72 & 56,57 & 55,32 \\
Conc. de sólidos em volume & $\%$ & 11,39 & 11,81 & 27,85 & 27,00 \\
Velocidade do rotor & $\mathrm{rpm}$ & 1500 & 1125 & 1250 & 1000 \\
\hline
\end{tabular}

Como se pode observar na Tabela 2, os ensaios 1 e 2 foram realizados com uma concentração de sólidos menor (30\%) e os ensaios 3 e 4 com uma concentração de sólidos maior (55\%). Por outro lado os ensaios 1 e 3 foram realizados com uma carga de corpos moedores menor (75\%) e os ensaios 2 e 4 com uma carga maior (80\%). A velocidade do rotor foi específica para cada ensaio, variando entre 1000 e 1500 RPM.

As distribuições de tempo de residência da fase líquida foram medidas através de uma injeção de ácido na mangueira de alimentação, gerando um pulso de traçador, neste caso $\mathrm{H}^{+}$. Os íons hidrogênio se misturam à água e viajam através do moinho na mesma velocidade da água. Os valores de $\mathrm{pH}$ foram medidos online com um pHmetro convencional instalado na mangueira do produto do moinho. O sistema determina o horário da medição, com isso as distribuições de tempo de residência podem ser estimadas. 
Os valores de $\mathrm{pH}$ podem ser convertidos para atividade de $\mathrm{H}^{+}$da seguinte forma:

$$
a H^{+}=1 / 10^{p H}
$$

A atividade de $\mathrm{H}^{+}$é proporcional à concentração de íons $\mathrm{H}^{+}$e pode ser diretamente empregada como se fosse a concentração de íons $\mathrm{H}^{+}$em solução. A principal vantagem deste sistema é a simplicidade com que as medidas podem ser feitas, sem a necessidade de análises químicas ou outros métodos indiretos de determinação da concentração do traçador. Outra vantagem é a medição instantânea correspondente a um tempo $t$ arbritário, sem a necessidade de integrar o tempo de recolhimento de amostra. Com isso a distribuição da atividade de íons $\mathrm{H}^{+}$em função do tempo $a \mathrm{H}^{+}(t)$ é dada por:

$$
a H^{+}(t)=K \sum_{i=1}^{N} \frac{\tau_{i}^{N-2} \exp \left(\frac{t_{d}-t}{\tau_{i}}\right)}{\prod_{k=1}^{N}\left(\tau_{i}-\tau_{k}\right)}, \quad i \neq k
$$

para $N$ misturadores perfeitos em série. $N a$ eq. (2) $\tau_{\mathrm{i}}$ é o tempo de residência do iésimo misturador perfeito na série, $t_{d}$ é o tempo correspondente ao transporte em fluxo pistão e $K$ é uma constante de proporcionalidade (SCHNEIDER, 1995). A variável $t_{d}$ deve ser necessariamente incluída porque o traçador é injetado em uma mangueira e é transportado até o misturador por fluxo pistão (plug flow). Isso se repete na mangueira de saída.

\section{RESULTADOS E DISCUSSÃO}

Os valores de tempo de residência foram calculados com base nas vazões volumétricas de sólidos e água e nos valores de holdup medidos, conforme apresentados na Tabela 3.

Tabela 3: Quantidades derivadas e medidas nos ensaios de moagem

\begin{tabular}{cccccc}
\hline Variável & Unid. & Teste 1 & Teste 2 & Teste 3 & Teste 4 \\
\hline Holdup de polpa & $\mathrm{cm}^{3}$ & 440,0 & 416,0 & 440,0 & 416,0 \\
Holdup de água & $\mathrm{cm}^{3}$ & 419,8 & 393,8 & 395,9 & 370,2 \\
Holdup de sólidos & $\mathrm{cm}^{3}$ & 16,6 & 18,3 & 40,5 & 41,9 \\
Holdup de sólidos & $\mathrm{kg}$ & 0,056 & 0,062 & 0,137 & 0,141 \\
Tempo de residência (sólidos) & $\mathrm{s}$ & 18,75 & 19,71 & 18,55 & 21,03 \\
Tempo de residência (água) & $\mathrm{s}$ & 60,39 & 55,73 & 70,07 & 68,27 \\
P80 & $\mu \mathrm{m}$ & 22 & 19 & 38 & 28 \\
Razão de redução & - & 4,05 & 4,68 & 2,34 & 3,18 \\
\hline
\end{tabular}

Os parâmetros de distribuição de tempos de residência são calculados interpolando-se as medições com o modelo da eq. (2). A melhor interpolação é obtida minimizando-se a soma dos quadrados das diferenças entre os valores calculados e medidos (Erro). Os valores de $\tau$ mostrados na Tabela 4 apresentam boa correlação com os valores de holdup de água calculados a partir do volume útil do moinho, descontados os volumes de sólidos e de bolas. 
Tabela 4: Parâmetros de distribuição de tempos de residência para o caso de três misturadores em série

\begin{tabular}{ccccc}
\hline Parâmetros & Teste 1 & Teste 2 & Teste 3 & Teste 4 \\
\hline$K$ & $7,45 \mathrm{E}-01$ & $8,96 \mathrm{E}-01$ & $5,44 \mathrm{E}-01$ & $6,22 \mathrm{E}-01$ \\
$\tau_{1}$ & 8,171 & 7,426 & 7,061 & 5,680 \\
$\tau_{2}$ & 8,180 & 7,431 & 7,065 & 5,682 \\
$\tau_{3}$ & 8,173 & 7,433 & 7,065 & 5,682 \\
$t_{d}$ & 26,948 & 27,315 & 29,048 & 31,718 \\
$\tau=\tau_{1+} \tau_{2} \tau_{3}$ & 24,524 & 22,290 & 21,191 & 17,044 \\
Total $=\tau+t_{d}$ & 51,473 & 49,605 & 50,240 & 48,763 \\
Erro & 0,473679 & 1,088453 & 0,30844 & 0,395862 \\
\hline
\end{tabular}

Por outro lado não há correlação entre os tempos de residência estimados por meio das vazões e dos holdups com os tempos de residência obtidos a partir das distribuições de tempos de residência medidos pela adição de ácido como traçador. Isso indica claramente que as estimativas de vazão não são acuradas. As estimativas de vazão deverão ser verificadas cuidadosamente em ensaios futuros. Mesmo que os tempos de residência medidos na DTR (Distribuição de Tempos de Residência) correlacionem bem com os volumes de holdup de água, a indicação é de que todos os procedimentos e estimativas sejam revisados. Por exemplo, não há como justificar o intercepto em -37.805 na Figura 3.

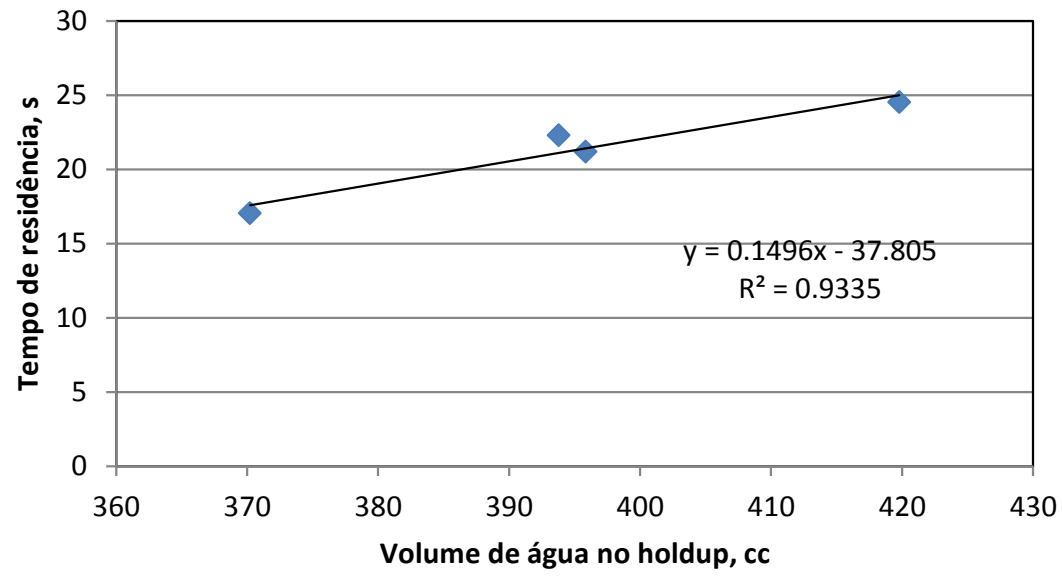

Figura 3: Correlação entre tempos de residência calculados a partir da eq. (2) $\operatorname{com~} \mathbf{N}=3$ e valores de volume de holdup de água.

As DTR medidas e interpoladas são mostradas na Figura 4 para os quatro testes realizados. A qualidade das interpolações é boa, mas podem ser melhoradas aumentando-se o número de misturadores em série. Os resultados dos ensaios foram analisados para três misturadores em série. Em geral os resultados mostram três misturadores com tempos de residência muito similares. Isso corresponde a três volumes idênticos de mistura. 

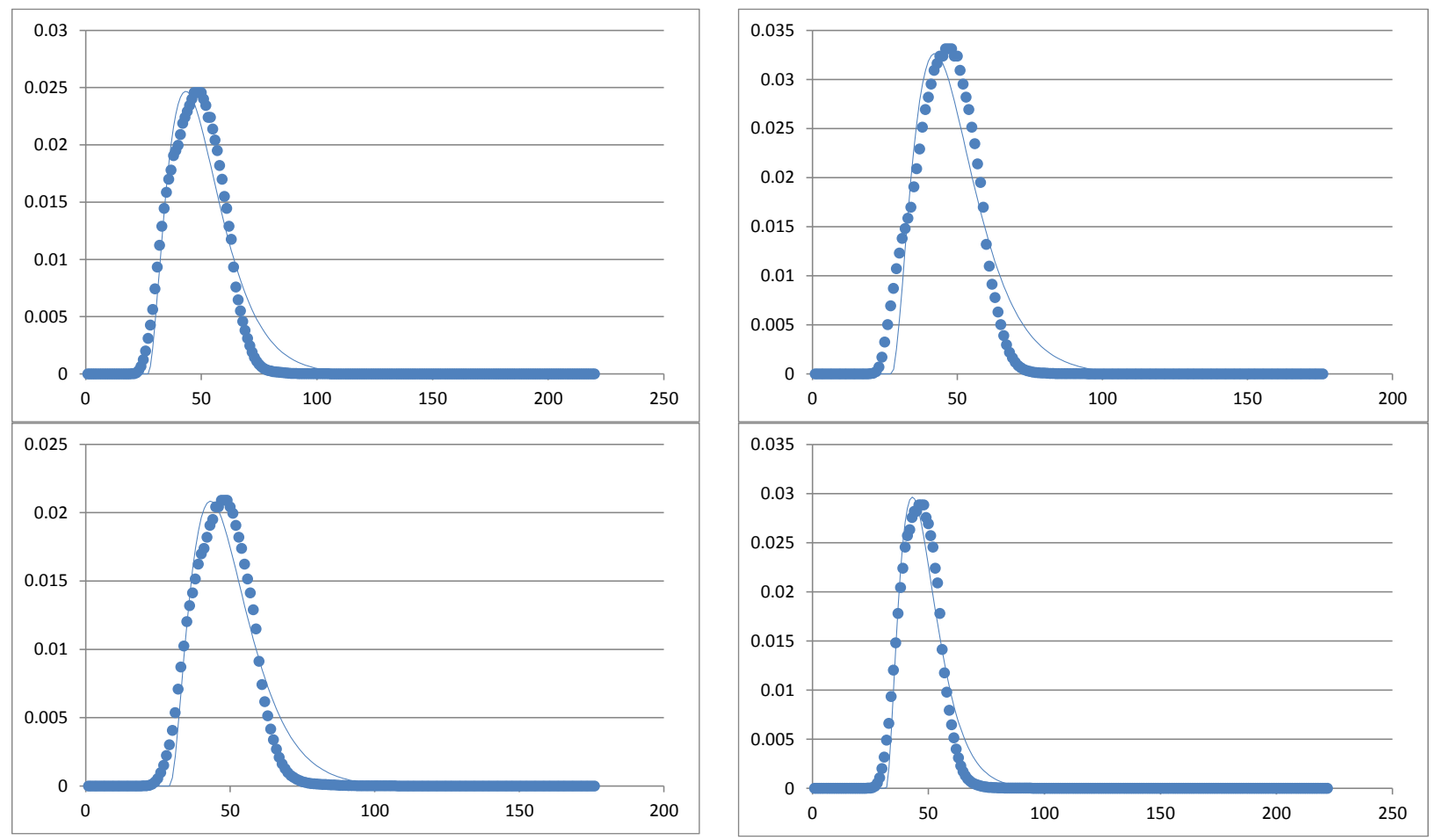

Figura 4: DTRs medidas (símbolos) e calculadas (linhas) para três misturadores em série. Ensaio1 no quadrante 1 e assim por diante. Eixo y mostra valores de atividade de íons $\mathrm{H}^{+}$e eixo $\mathrm{x}$ tempo, em segundos.

\section{CONCLUSÃO}

O sistema de determinação de distribuições de tempos de residência parece funcionar muito bem. O moinho IsaMill de laboratório apresenta um padrão de DTR de fluxo pistão, e deverá se comportar similarmente a um moinho de barras do ponto de vista de transporte. Classificação de saída não é considerada neste tipo de transporte, facilitando o trabalho de modelamento.

Os ensaios podem ser realizados de forma contínua ou em batelada, variando-se a taxa de alimentação no primeiro caso ou o tempo de moagem. Em função das dificuldades com medidas de vazão, recomenda-se que sejam realizados ensaios em batelada.

\section{REFERÊNCIAS BIBLIOGRÁFICAS}

1. BOSSE, D.G. Ultrafine grinding equipment types, capabilities and choices. in: Malghan, S. (Editor), Ultrafine grinding and separation of industrial minerals., Soc. Mining Engrs. Amer. Inst. Min. Metall. Pet. Engrs., New York, pp. 3-8, 1983.

2. KALRA, R. Overview on alternative methods for fine and ultra-fine grinding, In: IIR Conference, Crushing \& Grinding, Perth, 1999.

3. KWADE, A. Wet comminution in stirred media mills-research and its practical application, Powder Technol. 105, pp.14-20, 1999.

4. KWADE, A. Determination of the most important grinding mechanism in stirred media mills by calculating stress intensity and stress number, Powder Technol. 105, pp. 382-388, 1999.

5. KWADE, A \& SCHWEDES, J. Breaking characteristics of different materials and their effect on stress intensity and stress number in stirred media mills, Powder Technol. 122, pp. 109-121, 
2002.

6. HOGG, R. Breakage mechanisms and mill performance in ultrafine grinding, Powder Technol. 105, pp. 135-140, 1999.

7. JAYASUNDARA, C.T., YANG, R.Y., YU, A.B. Effect of the size of media on grinding performance in stirred mills, Minerals Engineering, Vol.33, pp. 66-71, 2012.

8. MANKOSA, M.J., ADEL, G.T., YOON, R.H. Effect of media size in stirred ball mill grinding of coal, Powder Technol. 49, pp. 75-82, 1986.

9. SCHNEIDER, C.L., Measurement and Calculation of Liberation in Continuous Milling Circuits, Ph.D. Dissertation, University of Utah, 1995.

10. SHINOHARA, K. Fine grinding characteristics of hard materials by attrition mill, Powder Technol. 103, pp. 292-296, 1999.

11. STEHR, N., MEHTA, R.K., HERBST, J.A. Comparison of energy requirements for conventional and stirred ball milling for coal - water slurries, Coal Preparation, Vol. 4, pp. 209-226, 1987.

12. YUE, J., KLEIN, B. Particle breakage kinetics in horizontal stirred mills, Minerals Engineering Vol.18, pp.325-331, 2005. 